\title{
A CLASS OF HYPERRINGS AND HYPERFIELDS
}

\author{
MARC KRASNER \\ Université de Paris VI \\ Paris, France 75006 \\ (Received March 1, 1982)
}

ABSTRACT. Hyperring is a structure generalizing that of a ring, but where the addition is not a composition, but a hypercomposition, i.e., the sum $x+y$ of two elements, $\mathrm{x}, \mathrm{y}$, of a hyperring $\mathrm{H}$ is, in general, not an element but a subset of $\mathrm{H}$. When the non-zero elements of a hyperring form a multiplicative group, the hyperring is called a hyperfield, and this structure generalizes that of a field. A certain class of hyperfields (residual hyperfields of valued fields) has been used by the author [1] as an important technical tool in his theory of approximation of complete valued fields by sequences of such fields. The non-commutative theory of hyperrings (particularly Artinian) has been studied in depth by Stratigopoulos [2] .

The question arises: How common are hyperrings? We prove in this paper that a conveniently defined quotient R/G of any ring $R$ by any normal subgroup $G$ of its multiplicative semigroup is always a hyperring which is a hyperfield when $R$ is a field. ive ask: Are all hyperrings isomorphic to some subhyperring of a hyperring belanging to the class just described?

KEY WORDS AND PHRASES. Hyperring, hyperfield.

1980 MATHEMATICS SUBJECT CLASSIFICATION CODE. 16 A78.

In 1956, in order to define a certain approximation of a complete valued field by sequences of such fields, I introduced in [I] the following structure $(F ; x+y, x y)$ that I called hyperfield: the multiplication $(x, y) \rightarrow x y$ is a composition of $F$, that is, a mapping of $\mathrm{F} \times \mathrm{F}$ into $\mathrm{F}$. But the addition $(\mathrm{x}, \mathrm{y}) \rightarrow \mathrm{x}+\mathrm{y}$ is a hypercomposition of $\mathrm{F}$, 
that is, a mapping of $F \times F$ into $P(F)$, the set of all subsets of $F$. According to established usage this hypercomposition can be extended to subsets of $\mathrm{F}$ as follows: $X+Y=U(x+y) \quad x \in X, y \in Y, X+Y=\{x\}+Y$, and $X+Y=X+\{y\}$. Finally, the structure satisfies the following axioms:

I. MULTIPLICATTVE AXIOM.

$F$ is a semigroup with respect to its multiplication and has a bilaterally absorbing element, denoted $0 ; \mathrm{F}-\{0\}$ is a group, always with respect to multiplication, whose neutral element is denoted 1.

II. ADDITIVE AXIOMS.

1. $\mathrm{x}+\mathrm{y}=\mathrm{y}+\mathrm{x}($ commutativity) ;

2. $(x+y)+z=x+(y+z)$ (associativity);

3. For every $x \in F$, there exists one and only one $x^{\prime} \in F$ such that $0 \in x+x^{\prime}$ (existence of the opposite element; such $x^{\prime}$ will be denoted $-\mathrm{x}$ and $\mathrm{x}-\mathrm{y}$ will be written instead $\mathrm{x}+(-\mathrm{y}))$;

4. $z \in x+y$ implies $y \in z-x$ (almost subtraction).

III. AXIOM OF DISTRIBUTIVITY. $z(x+y)=z x+z y,(x+y) z=x z+y z$.

The particular cases of this structure, which were used in [1], were the residual hyperfields of a valued field. Let $k$ be such a field and |..| its valuation. Let $i=\{x \in k ;|x| \leq 1\}$ be its valuation ring and $q$ be some proper ideal of $i$. Then $1+q$ is a multiplicative, nomal subgroup of $\mathrm{k}$ and its multiplicative classes, $\vec{x}=x(1+q) \quad(x \in k)$, form a partition of $k$. Let $k_{q}=k / 1+q$ be the set of these classes. Since $q$ is proper, if $z \in q$, then $|z|<1$. Thus all elements in class $\bar{x}$, have the same valuation, $|x|$, which will also be denoted $|\bar{x}|$ and called the valuation of $\bar{x}$. Clearly, if we multiply $\bar{x}, \bar{y} \in k_{q}$ as subsets of $k$, we have $\bar{x} \bar{y}=\overline{x y}$. Then, the multiplication as elements of $\mathrm{k}_{\mathrm{q}}$ will be the same, and $\mathrm{k}_{\mathrm{q}}$ will become a multiplicative almost-group. When we add $\bar{x}$ and $\bar{y}$ as subsets of $k$, their sum is generally not a single element $\bar{z}$ of $k_{q}$, but a union of such elements. And, if we define the sum of $\bar{x}$ and $\bar{y}$ in $k_{q}$ as the set of all $\bar{z}, z \in k$, contained in their sum as subsets of $k$, it can be verified that the obtained structure is a hyperfield, called the residual 
hyperfield of $k$ (mod $\left.{ }^{x} q\right)$. We defined in [1] an approximation of valued fields in the following manner: a complete valued field $k$ is approximated by a sequence $\mathrm{k}_{1}, \mathrm{k}_{2}, \ldots, \mathrm{k}_{\mathrm{s}}, \ldots$ of valued fields if, for each $\mathrm{s}$, is given an ideal $\mathrm{q}_{\mathrm{s}}$ of the valuation ring $i$ of $k$ and an ideal $q_{s}^{\prime}$ of the valuation ring $i_{s}$ of $k_{s}$ such that $\cap_{q_{s}}=0$ and the residual hyperfields $\bar{k}^{(s)}=k /\left(1+q_{s}\right)$ and $\bar{k}_{s}=k_{s} /\left(1+q_{s}^{\prime}\right)$ would be isomorphic with respect to their addition, multiplication and valuation. When the $k_{s}$ are also complete and, for each $s$, an isomorphism $u_{s}: \bar{k}_{s} \bar{k}^{(s)}$ is fixed, it is shown in [1] that, for each finite algebraic extension $k / k$ and for every $s$, since some index $s_{O}(K)$, such an extension $\mathrm{K}_{\mathrm{S}} / \mathrm{k}_{\mathrm{S}}$ can be almost canonically (to an automorphism of algebraic closure of $\mathrm{K}_{\mathrm{s}} / \mathrm{k}_{\mathrm{s}}$ ) defined, which is normal if and only if $\mathrm{K} / \mathrm{K}$ is normal and that the Galois groups $\mathrm{G}(\mathrm{K} / \mathrm{K})$ and $\mathrm{G}\left(\mathrm{K}_{\mathrm{S}} / \mathrm{k}_{\mathrm{S}}\right)$ are isomorphic.

I introduced later a more general structure $(H ; x+y, x y)$, which is in the same relation to hyperfields as rings are to fields. I called this structure hyperring. In this structure, the multiplication is still a composition, and the addition a hypercamposition on $\mathrm{H}$, which satisfy the same additive axiams II 1,2,3,4 and distributive axiom III as that of hyperfields, but the multiplicative axiom I is replaced by:

I'. $\mathrm{H}$ is a multiplicative semigroup having a bilaterally absorbing element 0 . A subset $h$ of a hyperring $H$ is said to be its subhyperring, if it is a hyperring with respect to the addition and multiplication induced by that of $\mathrm{H}$. It is easy to see that such is the case, if and only if, $h$ is closed under addition, multiplication ( $h+h \subseteq h, h h \subseteq h$ ), and opposition ( $x \in h$ implies $-x \in h$ ). My student, D. Stratigopoulos, has studied, in his thesis of the University of Louvain [2] , the structure of hyperrings in some detail, particularly in the conveniently defined Artinian case. He showed that, in spite of its seemingly irregular addition, some unexpectedly precise results can be proved about this structure (in particular about its idempotent elements and on the structure of primitive and simple hyperrings), the proofs of these results being quite hidden and far from trivial. The question arises: how cammon are these structures? It appears that they are very common and strongly related to such classical structures as rings, fields, and groups, as shown in the 
following theorem.

THEOREM. Let $\mathrm{R}$ be a ring and $\mathrm{G}$ a normal subgroup of its multiplicative semigroup (a subgroup $G$ of a semigroup $S$ is called normal if, for every $x S$, $x G=G x$ ). Then, the multiplicative classes $\bar{x}=x G(x \in R)$ form a partition of $R$, and let $\bar{R}=R / G$ be the set of these classes. The product of $\bar{x}, \bar{y} \in \bar{R}$ as subsets of $R$ is again a class $(\bmod G)$, and their sum as such subsets is a union of such classes. If we define the product $\bar{x} \bar{y}$ in $\bar{R}$ of $\bar{x}, \bar{y} \in \bar{R}$ as equal to their product as subsets of $R$, and their sum $\bar{x}+\bar{y}$ in $\bar{R}$ as the set of all $\bar{z} \in \bar{R}$ contained in their sum as subsets of $R$, the obtained structure is a hyperring (we call it the quotient hyperring of $R$ by $G)$. If $R$ is a field, $R / G$ is a hyperfield.

PROOF. As we have in $R$ (G being normal) $\bar{x} \bar{y}=x G y G=x y G G=x y G=\overline{x y}, x \rightarrow \bar{x}$ is a multiplicative homomorphism of $R$ onto $\bar{R}$, so $\bar{R}$ is a multiplicative semigroup. We have $\overline{0} \bar{x}=\overline{O x}=\overline{0}$ and $\bar{x} \overline{0}=\bar{x} 0=\overline{0}$, so $\overline{0}$ is bilaterally absorbing. If $R$ is a multiplicative almost-group, i.e., $R=R^{\star} U\{0\}$ where $R^{\star}$ is a group, and if 1 is the neutral element of $R^{*}$, we have, for every $x \in R^{*}, \bar{x} \overline{x^{-1}}=\overline{x^{-1}} \bar{x}=\bar{I}$, and $\bar{R}$ is also a multiplicative almost-group.

Let $x, y$ be in $R$ and let $\bar{x}+\bar{y}$ denote the sum of $\bar{x}, \bar{y}$ as subsets of $R$. Let $z$ be an element of $\bar{x}+\bar{y}$. Then, there exist $x^{\prime} \in \bar{x}$ and $y^{\prime} \in \bar{y}$ such that $z=x^{\prime}+y^{\prime}$. But, as $G$ is a group, $x^{\prime} \in X G$ implies $\overline{x^{\prime}}=x^{\prime} G=x G=\bar{x}$, and also $y^{\prime} \in \bar{y}$ implies $\overline{y^{\prime}}=\bar{y}$. But then $\bar{z}=z G=\left(x^{\prime}+y^{\prime}\right) G \leqq x^{\prime} G+y^{\prime} G=\bar{x}+\bar{y}$. So $\bar{x}+\bar{y}$ is a union of classes (mod $G$ ), and by definition, $\bar{x}+\bar{y}=\{\bar{z} \in \bar{R} ; \bar{z} \subseteq \bar{x}+\bar{y}\}$.

As the addition in $R$ is commutative, we have obviously $\bar{x}+\bar{y}=\bar{y}+\bar{x}$. We have

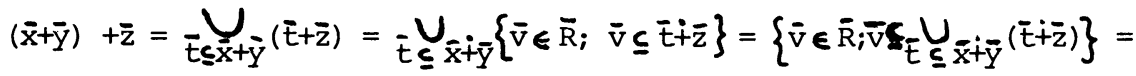
$\{\bar{v} \in \bar{R} ; \bar{v} \subseteq(\bar{x}+\bar{y})+\bar{z}\}$. We prove in an analogous way that $\bar{x}+(\bar{y}+\bar{z})=\{\bar{v} \in \bar{R} ; \bar{v} \leq \bar{x} \dot{+}(\bar{y}+\bar{z})\}$. Since the addition of subsets of $R$ is associative, we have $(\bar{x}+\bar{y})+\bar{z}=\bar{x} \dot{+}(\bar{y}+\bar{z})$ and $(\bar{x}+\bar{y})+\bar{z}=\bar{x}+(\bar{y}+\bar{z})$.

Suppose that $\overline{0} \in \bar{x}+\bar{y}$, i.e., $0 \in \bar{x}+\bar{y}$. That is equivalent to the existence of an $x^{\prime} \in \bar{x}$, such that $-x^{\prime} \in \bar{y}$. But then, $\bar{y}=\overline{-x^{\prime}}=\left(-x^{\prime}\right) G=-x^{\prime} G=-\overline{x^{\prime}}=-\bar{x}$. So, the only $\bar{y} \in \bar{R}$ such that $\overline{0} \in \bar{x}+\bar{y}$ is $\bar{y}=\overline{-x}=-x G$.

Finally, $\bar{z} \in \bar{x}+\bar{y}$ is equivalent to $\bar{z} \subseteq \bar{x}+\bar{y}$. So there exist an $x^{\prime} \in \bar{x}$ and a $y^{\prime} \in \bar{y}$ 
such that $z=x^{\prime}+y^{\prime}$, and $y^{\prime}=z+\left(-x^{\prime}\right)$. So we have $y=\overline{y^{\prime}}=\overline{z+\left(-x^{\prime}\right)} \subseteq \bar{z}+\left(\overline{-x^{\prime}}\right)=$ $\bar{z} \dot{+}(\overline{-x})$ and $\bar{y} \in \bar{z}-\bar{x}$.

We have $\bar{z}(\bar{x}+\bar{y})=\{\bar{z} \bar{t} ; \bar{t} \subseteq \bar{x}+\bar{y}=x G+y G\}$. But $\bar{z} \bar{t}=\overline{z t}=z t G=z \bar{t}$ and $\bar{t} \subseteq x G+y G \Rightarrow z \bar{t} \subseteq z(x G \dot{+} y G)=z x G+z y G=\overline{z x}+\overline{z y}=\bar{z} \bar{x}+\bar{z} \bar{y}$. On the other hand, if $\bar{v} \subseteq \bar{z} \bar{x}+\bar{z} \bar{y}=z(x G \dot{+} y G)$, we have $v \in z(x G \dot{y} G)$, so $v=z t$, where $t \in x G \dot{+} y G=\bar{x}+\bar{y}$ and $\bar{v}=\bar{z} \bar{t}$, where $\bar{t} \in \bar{x}+\bar{y}$. So, $\bar{v} \in \bar{z}(\bar{x}+\bar{y})$ is equivalent to $\bar{v} \subseteq \bar{z} \bar{x} \dot{x} \bar{z} \bar{y}$, i.e., to $\bar{v} \in \bar{z} \bar{x}+\bar{z} \bar{y}$, and we have $\bar{z}(\bar{x}+\bar{y})=\bar{z} \bar{x}+\bar{z} \bar{y}$. We prove in a similar manner that $(\bar{x}+\bar{y}) \bar{z}=\bar{x} \bar{z}+\bar{y} \bar{z} . \quad$ All is proved.

abviously, G is a bilateral neutral element of the multiplicative semigroup of $\bar{R}=R / G$, and as it is well known, that implies that this semigroup has no other left or right neutral element. A subset $S$ of $R$ is a union of classes (mod G) if, and only if, $S G=S$. If $\bar{S}=S / G$ is the set of these classes, then it is clear that $\bar{S}$ is a subhyperring of $\bar{R}$, if and only if $S$ is a subring of $R$, and such $\bar{S}$ are the only subhyperrings of $\overline{\mathrm{R}}$.

The question arises: Are all hyperrings which are not rings isomorphic to the subhyperrings of quotient hyperrings $R / G$ of some ring $R$ by some of its normal multiplicative subgroups $G$ when they are not rings? Are all hyperfields isomorphic to a quotient $k / G$ of a field $k$ by some of its multiplicative subgroups $G$ ?

\section{REFERENCES}

1 Krasner, M. Approximation des corps valués complets de caractéristique $p \neq 0$ par ceux de caractéristique 0. Colloque d'Algèbre Supérieure (Bruxelles, décembre 1956), CBRM, Bruxelles, 1957.

2 Stratigopoulos, D. Hyperanneaux artiniens (Thèse de doctorat de l'Université de Louvain, mimeographiée). 


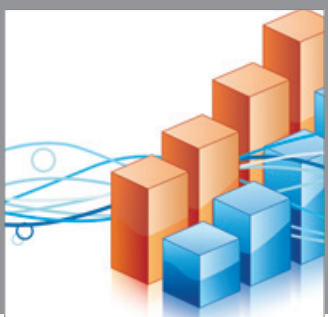

Advances in

Operations Research

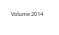

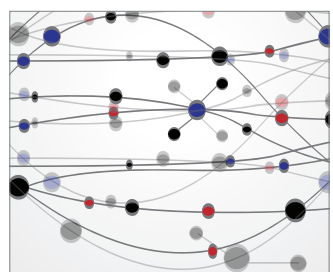

\section{The Scientific} World Journal
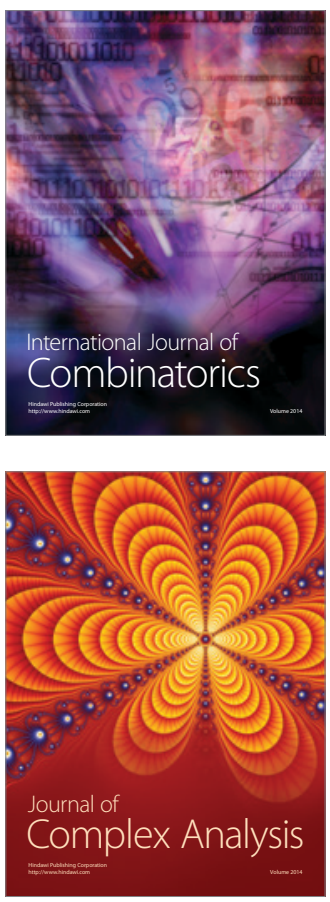

International Journal of

Mathematics and

Mathematical

Sciences
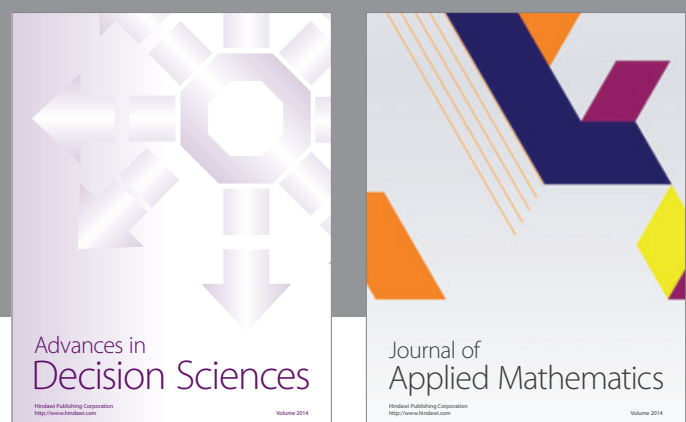

Journal of

Applied Mathematics
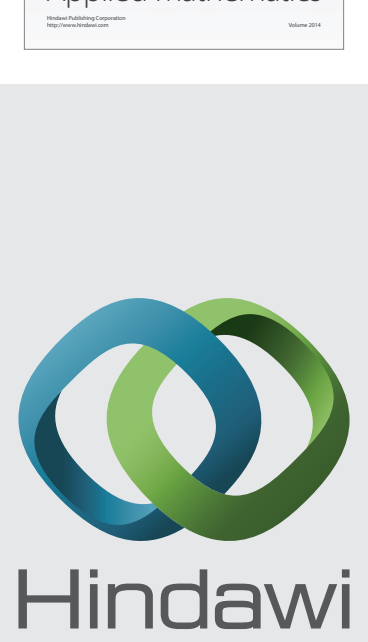

Submit your manuscripts at http://www.hindawi.com
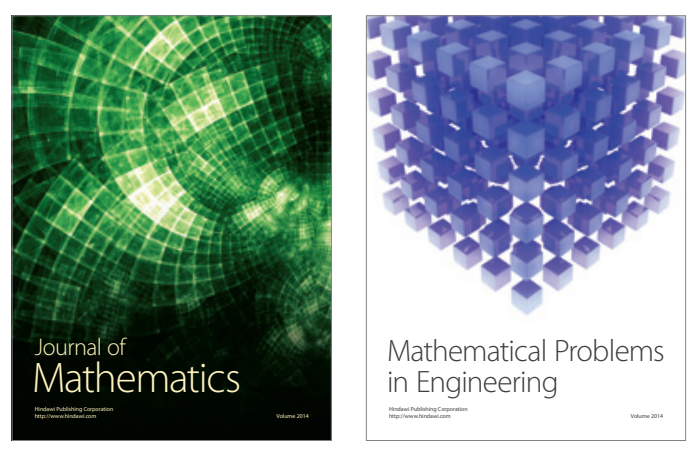

Mathematical Problems in Engineering
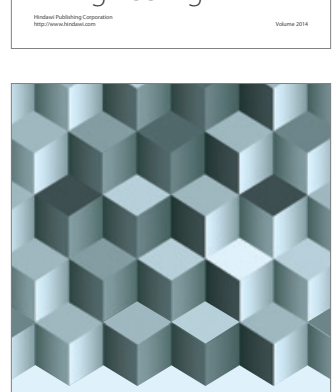

Journal of

Function Spaces
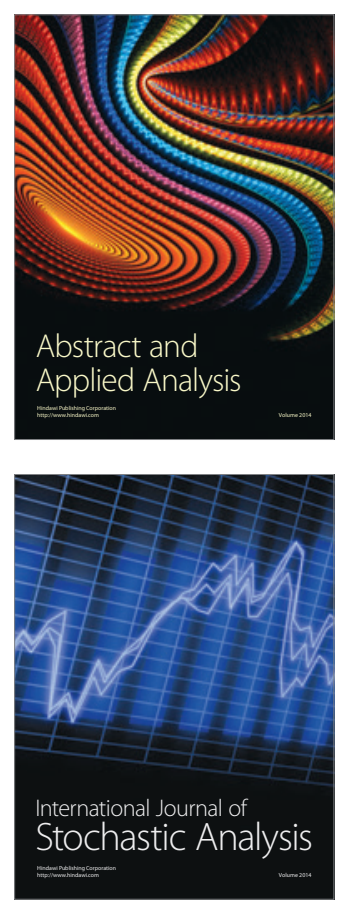

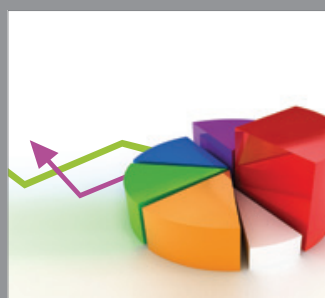

ournal of

Probability and Statistics

Promensencen
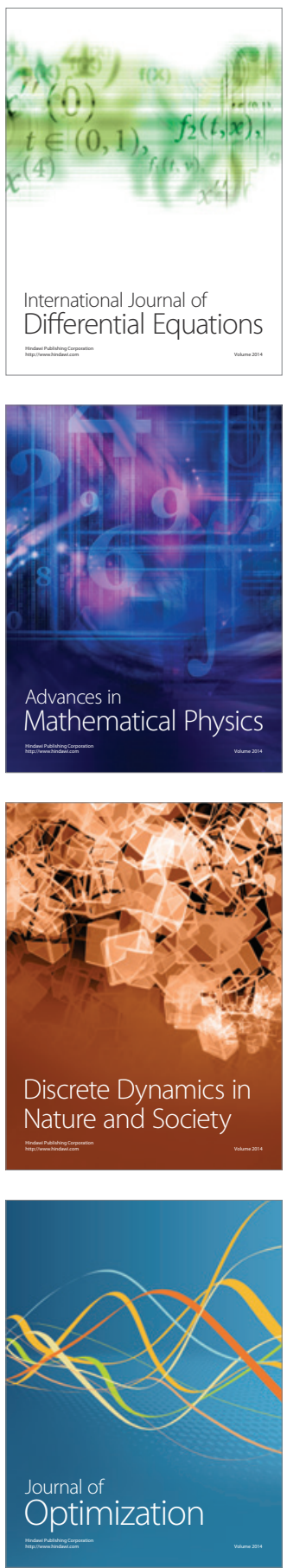